\title{
Tumores estromales gastrointestinales (GIST) gástricos, serie de casos
}

\section{Gastrointestinal stromal tumors (GIST). Case series}

\author{
Ricardo Oliveros,,$^{1^{*}}$ (1) Raúl Pinilla, ${ }^{1}$ (1] Ricardo Sánchez, ${ }^{2}$ [0 Haylmanrth Contreras. ${ }^{1}$
}

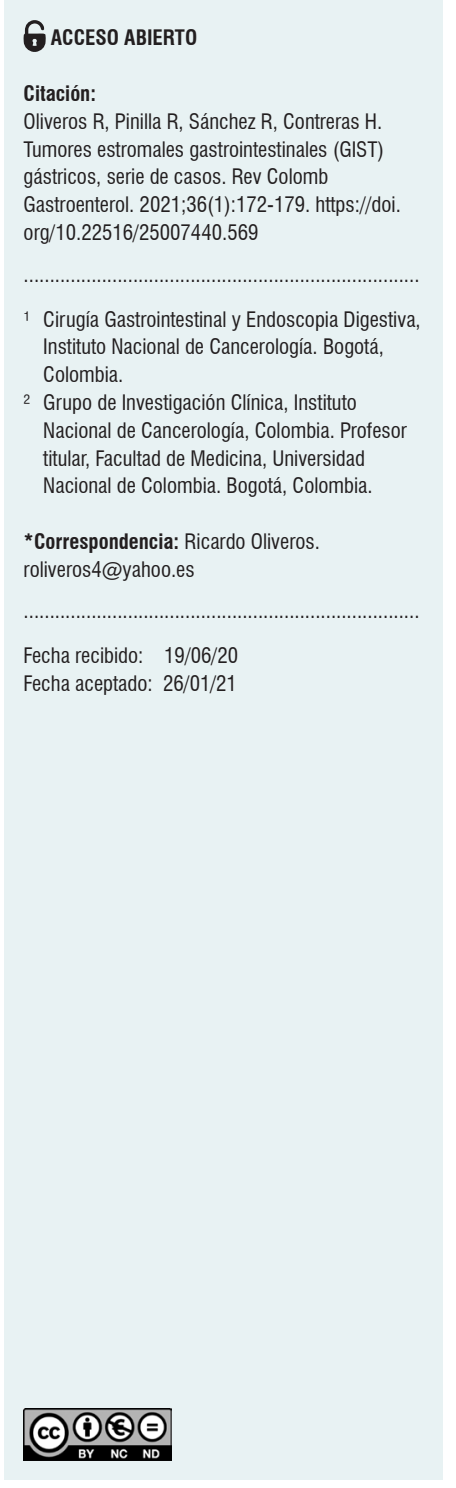

\section{Resumen}

Introducción: los tumores estromales gastrointestinales (GIST) representan hasta el $2 \%$ de las neoplasias gastrointestinales, estos aparecen en cualquier parte del tracto gastrointestinal y son encontrados más frecuentemente en el estómago (60 \%). El diagnóstico se realiza por la expresión de un receptor de factor de crecimiento de tirosina-cinasa, antígeno de diferenciación (CD) 117, lo que lo diferencia de los otros tumores mesenquimales como leiomiomas, leiomiosarcomas, leiomioblastomas y tumores neurogénicos, que no expresan esta proteína. Objetivo: el objetivo de nuestro trabajo es caracterizar los GIST de localización gástrica con respecto a su presentación clínica, diagnóstico, manejo, recurrencia y supervivencia. Métodos: se trata de un estudio observacional, retrospectivo basado en una serie de casos. Se realizó una extracción de la información por medio de la revisión de las historias clínicas de los pacientes con GIST gástricos en un centro oncológico de Bogotá entre enero de 2005 y diciembre de 2015. La información recolectada incluyó tipo de manejo y abordaje quirúrgico, localización, tamaño, índice mitótico y clasificación de riesgo. Resultados: se encontraron 31 pacientes con diagnóstico de GIST gástrico. La edad media fue de 62,3 años, con una mediana de 61 años. De los 31 pacientes, 18 fueron mujeres y 13 hombres. El tiempo de seguimiento estuvo entre un mínimo de 2,4 meses y un máximo de 214 meses, La mediana de seguimiento fue de 36 meses. Conclusiones: Ios GIST son tumores potencialmente malignos, y el de localización gástrica es el más frecuente. El diagnóstico y tratamiento dependen de su tamaño y localización dentro del estómago. El manejo es variado y consiste en la resección quirúrgica, en la que los procedimientos son mínimamente invasivos, en combinación con la endoscopia, que son una buena alternativa al tratamiento abierto hasta que haya necesidad de terapia sistémica.

\section{Palabras clave \\ Tumor estromal gastrointestinal (GIST) gástrico, manejo, localización.}

\section{Abstract}

Introduction: Gastrointestinal stromal tumors (GIST) account for up to $2 \%$ of gastrointestinal neoplasms. They may appear anywhere in the gastrointestinal tract and are most frequently found in the stomach (60\%). The diagnosis is made based on the expression of the tyrosine-protein kinase KIT CD117, which differentiates it from other mesenchymal tumors such as leiomyomas, leiomyosarcomas, leiomyoblastomas, and neurogenic tumors, that do not express this protein. Objective: To characterize the clinical presentation, diagnosis, treatment, recurrence, and survival of GISTs in the stomach. Materials and methods: This is an observational retrospective study based on a case series. Data was collected after reviewing the medical records of patients diagnosed with GIST at a cancer center in Bogotá between January 2005 and December 2015. The data included type of treatment and surgical approach, location, size, mitotic index, and risk classification. Results: There were 31 patients diagnosed with GIST. Their mean age was 62.3 years, with a median of 61 years. Of the 31 patients, 18 were women and 13 were men. Follow-up time ranged from a minimum of 2.4 months to a maximum of 214 months. The median follow-up time was 36 months. Conclusions: GISTs are potentially malignant tumors, with gastric location being the most frequent. Diagnosis and treatment depend on their size and location in the stomach. Treatment options vary, ranging from surgical resection, where minimally invasive procedures along with endoscopy are a suitable alternative to open surgery, to the need for systemic therapy.

\section{Keywords}

Gastrointestinal stromal tumor; Management; Localization. 


\section{INTRODUCCIÓN}

Los tumores estromales gastrointestinales (GIST) son los tumores mesenquimales más comunes del tracto gastrointestinal y representan del $1 \%$ al $2 \%$ de todos los tumores gastrointestinales (1). Se estima que la tasa de incidencia de los GIST es de 1 caso x 100000 habitantes por año. En los Estados Unidos, la incidencia varía de 5000 a 10000 casos por año (2).

Los GIST ocurren en cualquier parte del tracto gastrointestinal y se encuentran más frecuentemente en el estómago $(60 \%)$, seguido por el intestino delgado (30\%) y entre el $5 \%$ y el $10 \%$ en cualquier parte del tracto gastrointestinal (esófago, colon, recto, epiplón y mesenterio) (3). Se originan en las células intersticiales de Cajal, encargadas del marcapaso del tracto gastrointestinal, cuya mutación en el gen KIT parece ser la principal responsable del crecimiento de estos tumores. El diagnóstico del GIST está fundamentado en el conocimiento de las características macroscópicas e histológicas, y el espectro inmunohistoquímico (3).

Las lesiones de localización gástrica se presentan con un amplio rango de comportamiento clínico, que varía desde lesiones de pequeño tamaño descubiertas de manera casual hasta aquellos de gran tamaño con gran agresividad y capacidad de diseminación. Los síntomas no son específicos y dependen del tamaño y su localización. Muchas lesiones son pequeñas (menores de $2 \mathrm{~cm}$ ) y no causan síntomas, pero son descubiertas de forma incidental durante procedimientos endoscópicos, durante cirugías abdominales o en imágenes radiológicas. Estas lesiones también pueden causar molestias inespecíficas como dispepsia y emesis, y en ocasiones pueden presentarse con masa, dolor abdominal intenso e incluso sangrado digestivo (3).

El objetivo de nuestro trabajo es caracterizar los GIST de localización gástrica con respecto a su presentación clínica, diagnóstico, manejo, recurrencia y supervivencia.

\section{MATERIAL Y MÉTODOS}

La información se obtuvo por medio de la revisión de las historias clínicas de los pacientes con GIST gástricos en un centro oncológico de Bogotá, entre enero de 2005 y diciembre de 2015. Se registraron las características demográficas, clínicas, histológicas e inmunohistoquímicas en biopsia y espécimen quirúrgico de cada uno de los casos detectados. Dentro de los tratamientos efectuados se incluyeron el quirúrgico abierto y el manejo laparoscópico, además de la quimioterapia perioperatoria en algunos casos. La información acerca del procedimiento incluyó el tipo de manejo y el abordaje quirúrgico, localización, tamaño, índice mitótico y clasificación de riesgo. La categorización del riesgo fue efectuada con base en el consenso del National
Institute of Health (NIH) y del Instituto de Patología de Las Fuerzas Armadas de los Estados Unidos, en relación con el tamaño de la lesión y su índice mitótico (4).

El manejo con imatinib se realizó en varios escenarios, como adyuvancia después de la resección quirúrgica estándar en caso de lesiones resecables y clasificadas como de alto riesgo y con intención neoadyuvante por 3 a 6 meses, en caso de lesiones localmente avanzadas o con resecabilidad limítrofe. Las lesiones irresecables, metastásicas o recurrentes también recibieron tratamiento con imatinib. El seguimiento se realizó hasta su último control de forma clínica y por imágenes, con el que se establecía la supervivencia libre de enfermedad, supervivencia global, recurrencia y manejo de esta.

\section{ANÁLISIS ESTADÍSTICO}

El presente es un estudio observacional, retrospectivo basado en una serie de casos. Las variables categóricas fueron resumidas como porcentajes. En el caso de las variables continuas, se usaron como medida de resumen medianas junto con el correspondiente rango intercuartílico (RIC). Como desenlaces se consideraron la muerte y la recurrencia; para su descripción se estimaron tasas de densidad de incidencia, teniendo en cuenta el tiempo diferencial de seguimiento. Estas tasas se expresaron tomando como denominador 100 pacientes/año y se calcularon tomando las pérdidas de seguimiento o la no presencia de desenlace como censuras a la derecha; las tasas se calcularon junto con sus correspondientes intervalos de confianza (IC) del $95 \%$. Adicionalmente, se calcularon y graficaron las funciones de supervivencia usando el método de Kaplan-Meier. Los análisis estadísticos se realizaron con el programa Stata $12^{\circledR}$.

\section{RESULTADOS}

\section{Características demográficas y clínicas}

De enero de 2005 a diciembre de 2015 se encontraron 31 pacientes con diagnóstico de GIST gástrico. La mediana fue de 61 años (RIC: 12). De los 31 pacientes, 18 fueron mujeres y 13 hombres. En relación con la presentación clínica, el dolor abdominal fue el síntoma más reportado por los pacientes (Tabla 1).

De acuerdo con la localización en el estómago, el sitio más frecuente fue el cuerpo (Tabla 1).

3 casos asintomáticos se diagnosticaron de forma incidental por endoscopia digestiva alta y por imágenes tomográficas.

En relación con el tamaño del tumor, la lesión de mayor tamaño fue de $26 \times 20 \mathrm{~cm}$ y la de menor tamaño, de $2 \times 2 \mathrm{~cm}$. Cuando se relacionó el tamaño con el índice mitótico para 
estratificar el riesgo se encontraron 11 pacientes $(35,5 \%)$ de riesgo alto, 16 pacientes $(51,6 \%)$ de riesgo bajo y 4 pacientes de riesgo intermedio $(12,9 \%)$.

Tabla 1. Características demográficas y clínicas de los casos del estudio

\begin{tabular}{|c|c|c|}
\hline & $n$ & $\%$ \\
\hline $\begin{array}{l}\text { Población } \\
\text { - Masculino } \\
\text { - Femenino } \\
\text { - Total }\end{array}$ & $\begin{array}{l}18 \\
13 \\
31\end{array}$ & $\begin{array}{l}58,1 \\
41,9\end{array}$ \\
\hline $\begin{array}{l}\text { Síntomas } \\
\text { - Dolor } \\
\text { - Sangrado } \\
\text { - Masa } \\
\text { - Asintomático } \\
\text { - Total }\end{array}$ & $\begin{array}{c}15 \\
7 \\
6 \\
3 \\
31\end{array}$ & $\begin{array}{l}48,4 \\
22,6 \\
19,4\end{array}$ \\
\hline $\begin{array}{l}\text { Localización } \\
\text { - Cuerpo } \\
\text { - Antro } \\
\text { - Fondo } \\
\text { - Total }\end{array}$ & $\begin{array}{c}20 \\
6 \\
5 \\
31\end{array}$ & $\begin{array}{l}64,5 \\
19,4 \\
16,1\end{array}$ \\
\hline $\begin{array}{l}\text { Riesgo } \\
\text { - Alto } \\
\text { - Intermedio } \\
\text { - Bajo } \\
\text { - Total }\end{array}$ & $\begin{array}{c}11 \\
4 \\
16 \\
31\end{array}$ & $\begin{array}{l}35,5 \\
12,9 \\
51,6\end{array}$ \\
\hline $\begin{array}{l}\text { Cirugía } \\
\text { - Abierta } \\
\text { - Laparoscopia } \\
\text { - Ninguna } \\
\text { - Total }\end{array}$ & $\begin{array}{l}21 \\
6 \\
4 \\
31\end{array}$ & $\begin{array}{l}67,7 \\
19,4 \\
12,9\end{array}$ \\
\hline $\begin{array}{l}\text { Tipo histológico } \\
\text { - Fusocelular } \\
\text { - Epitelioide } \\
\text { - Mixto } \\
\text { - No informado } \\
\text { - Total }\end{array}$ & $\begin{array}{c}16 \\
2 \\
5 \\
8 \\
31\end{array}$ & $\begin{array}{c}51,6 \\
6,5 \\
16,1 \\
25,8 \\
16,1\end{array}$ \\
\hline $\begin{array}{l}\text { Inmunohistoquímica } \\
\text { - CD117 } \\
\text { - CD34 } \\
\text { - Total }\end{array}$ & $\begin{array}{c}28,0 \\
27,0 \\
31\end{array}$ & $\begin{array}{c}90,32 \\
87,1\end{array}$ \\
\hline $\begin{array}{l}\text { Mutación del exón } 11 \\
\text { - Mutado } \\
\text { - No mutado } \\
\text { - Total }\end{array}$ & $\begin{array}{c}2 \\
29 \\
31\end{array}$ & $\begin{array}{c}6,5 \\
93,5\end{array}$ \\
\hline
\end{tabular}

CD: antígeno de diferenciación.

\section{Características histológicas-inmunohistoquímicas}

Con respecto a la distribución histológica, el tipo fusocelular fue el más frecuente encontrado, en 16 pacientes, seguido por el tipo epitelioide, en 2 pacientes, mixto en 5 pacientes y sin registro en 8 pacientes. El CD117 fue posi- tivo en el 90,32 \% de los casos ( $\mathrm{n}=28), \mathrm{CD} 34$ en el 87,1 \% $(\mathrm{n}=27)$. El Ki-67 fue registrado en el $71 \%$ de los pacientes $(\mathrm{n}=22)$ con una mediana de 4,5 $(\mathrm{RIC}=3)$. El índice mitótico tuvo una mediana de $3 \times 50$ CAP $(\mathrm{RIC}=3) .5$ pacientes presentaron metástasis $(16,1 \%)$ que se localizaron en el hígado (en los 5 casos) y 2 en el peritoneo.

En relación con el estudio molecular o estudio mutacional, este se realizó en 2 pacientes en los cuales se detectó la mutación del exón 11.

\section{Tratamiento quirúrgico}

El tratamiento quirúrgico más frecuentemente realizado fue la cirugía abierta en 21 de los casos $(67,74 \%)$, seguido del tratamiento por laparoscopia en $6(19,35 \%)$ y no se practicó ningún procedimiento en 4 pacientes por el tamaño de la lesión ( 2 casos), por edad avanzada ( 1 paciente) y por enfermedad metastásica (1 paciente) (Tabla 1$)$.

\section{Manejo médico}

De los 31 pacientes, 16 recibieron imatinib (IMB), en 8 casos después de la resección quirúrgica (adyuvancia) por ser lesiones de alto riesgo, en 2 casos por tratarse de lesiones metastásicas y en los otros 3 con intención neoadyuvante; los 3 restantes la recibieron por recurrencia de la enfermedad. El tratamiento se realizó con una mediana de 0 meses $(\mathrm{RIC}=24)$. De los pacientes con terapia adyuvante, 3 recibieron tratamiento por 3 años, 3 por 1 año y 2 por 2 años.

\section{Seguimiento}

Se presentaron 3 casos de recurrencia. La mediana de seguimiento fue de 36 meses. La función de supervivencia se ilustra en las Figuras 1, 2 y 3 (más del $50 \%$ de los casos

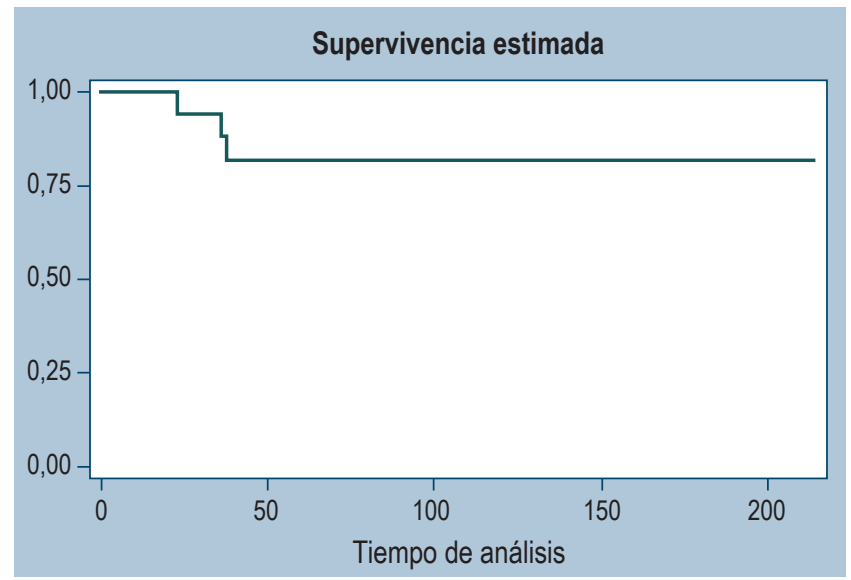

Figura 1. Función de supervivencia global para pacientes con GIST gástricos. 
no presentaron desenlace de mortalidad, por lo que no es estimable la mediana de supervivencia).

Hubo 2 casos de muerte y un total de 1480 meses aportados al seguimiento. Este tiempo de seguimiento estuvo entre un mínimo de 2,4 meses y un máximo de 214,7 meses. La mediana de seguimiento fue de 41,4 meses. La tasa de incidencia de mortalidad fue de 0,14 muertes por 100 pacientes/mes (IC $95 \%$ : 0,03 a 0,34).

De los 11 pacientes con riesgo alto, 6 pacientes se encontraban vivos a los 5 años, pero con enfermedad; es decir que, a pesar de tratarse pacientes con riesgo alto, más del $45 \%$ estaba vivo y sin enfermedad a los 3 años.

\section{DISCUSIÓN}

La verdadera incidencia de los GIST no ha sido bien establecida. Sin embargo, en los Estados Unidos la incidencia anual estimada es de 3000 a 6000 casos por año. Se pueden presentar a cualquier edad y la mayoría de los pacientes se encuentran entre los 40 a 80 años, y es un poco más frecuente en los hombres. Estas lesiones se pueden originar en cualquier parte del tracto gastrointestinal, y el estómago es el sitio más comprometido (5).

En la mayoría de las series, los tumores gástricos se encuentran entre el $45 \%$ y el $60 \%$ (6). Para Folgado y colaboradores, en una revisión de 43 pacientes durante 10 años, $20(46,5 \%)$ correspondían a localización gástrica (7). En una serie de 31 pacientes, Vargas y colaboradores encontraron 14, correspondientes al $45 \%$ de los casos (8). En una revisión del Instituto Nacional de Cancerología de Bogotá, del 2000 al 2008 se reportaron 39 pacientes, de los cuales

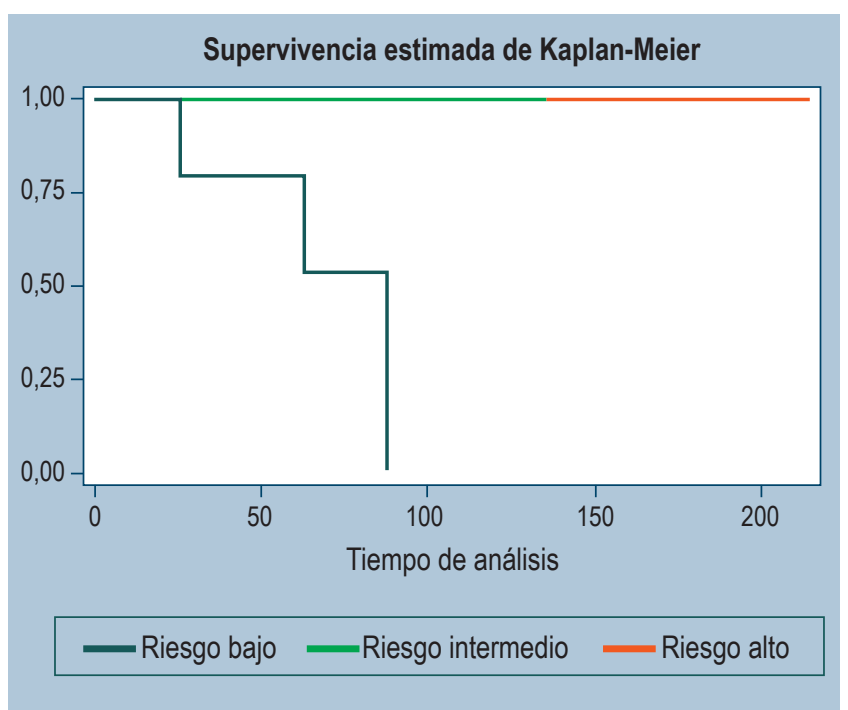

Figura 2. Función de supervivencia estratificada según el riesgo.
16 correspondían a localización gástrica (41\%) (9). Para Flores-Funes en España, en una revisión de 2002 a 2015 con 66 pacientes, 43 casos ( $65 \%)$ se encontraban localizados en el estómago (10).

Las manifestaciones clínicas varían ampliamente. El 70 \% de los pacientes cursa con síntomas, mientras que el $20 \%$ es asintomático y el $10 \%$ se descubre en estudios de autopsia (5). Para algunas series, la manifestación más frecuente fue la hemorragia digestiva ( $45 \%$ ), seguida del dolor abdominal (30\%) (11). En esta serie, el $80 \%$ de los pacientes presentó síntomas y el dolor abdominal fue el más frecuente en 18 pacientes, seguido por el sangrado digestivo en 7.3 pacientes cursaron asintomáticos $(9,7 \%)$ con lesiones de 2 $\mathrm{cm}$ en 2 pacientes y el tercero con una lesión de $4 \mathrm{~cm}$.

Los síntomas dependen mucho del tamaño. Los tumores mayores de $6 \mathrm{~cm}$ generalmente son sintomáticos, mientras que las lesiones menores de $2 \mathrm{~cm}$ son indolentes. En relación con el tamaño se encontró que la lesión de mayor tamaño correspondía a una de 26 × $20 \mathrm{~cm}$ y la de menor tamaño era de $2 \mathrm{~cm}$. Las lesiones mayores de $5 \mathrm{~cm}$ se encontraron en 17 pacientes y, cuando se relacionó el tamaño con el índice mitótico para estratificar el riesgo, se encontraron 11 pacientes $(35,5 \%)$ de riesgo alto, 16 pacientes $(51,6 \%)$ de riesgo bajo y 4 pacientes de riesgo intermedio $(12,9 \%)$.

En relación con la localización, el fondo parece ser el sitio predominante con el $25 \%$, seguido del cuerpo y, por último, del antro (10\%) (10).

En esta serie, el cuerpo fue el sitio de mayor número de lesiones (20 casos), seguido por el antro y el fondo.

La historia natural del GIST gástrico es variable y no ha sido completamente elucidada. Una de las características más

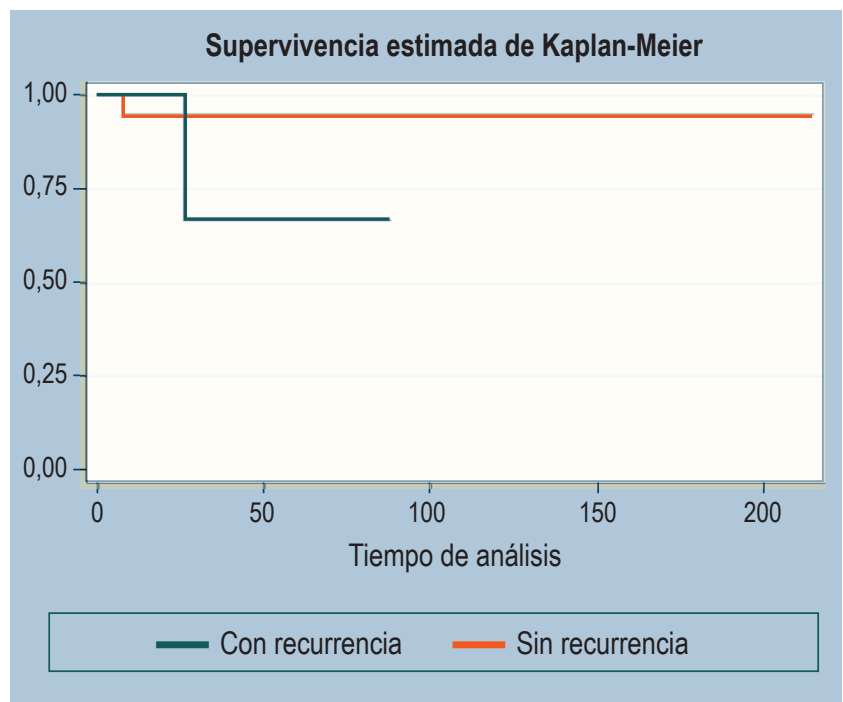

Figura 3. Función de supervivencia estratificada según la recurrencia. 
importantes es su variable e impredecible comportamiento biológico. No son definidos como malignos o benignos, pero su estratificación de riesgo de malignidad está basado en el tamaño de la lesión y el índice o conteo mitótico (12).

Las lesiones pequeñas tienen a tener un comportamiento biológico benigno, mientras que las lesiones de mayor tamaño son más agresivas; sin embargo, parece ser que toda lesión gástrica tiene el potencial de ser maligna. Por esta razón, la remoción quirúrgica es la recomendación una vez que se diagnostican. Varios estudios se han realizado para medir el curso natural de las lesiones no resecadas. Estos estudios limitan el tamaño de las lesiones subepiteliales a $2-3 \mathrm{~cm}$ y enfatizan cambios en el tamaño y ecoestructura determinada por la valoración endosonográfica en un período de seguimiento (12). Las lesiones gástricas tienen un pronóstico más favorable en comparación con las lesiones de otras localizaciones (13).

Estas lesiones son muy friables e hipervascularizadas, por lo que se visualizan como lesiones heterogéneas en las imágenes tomográficas. El diagnóstico es usualmente confirmado por examen patológico. La morfología corresponde en el $70 \%$ a una forma fusiforme, en el $20 \%$ a una forma epitelioide y en el $10 \%$ a una forma mixta (14); hallazgos similares a lo reportado en esta serie.

La proliferación de los GIST es producto de la mutación en el protooncogén KIT del receptor de tirosina-cinasa en el $80 \%$ o en el factor de crecimiento derivado de las plaquetas en el $10 \%$. El otro $10 \%$ corresponde a mutaciones del gen BRAF (14).

Todos expresan el CD117, la positividad del C-KIT es el marcador inmunohistoquímico más específico. Aproximadamente, el $5 \%$ de estos tumores pueden ser C-KIT negativos, CD34 + del $60 \%$ a $70 \%$, SM $+\operatorname{del} 30 \%$ a $40 \%$ y S 100 + en un $5 \%$. El DOG1 puede ser útil en casos de GIST con C-KIT negativo. Aproximadamente, el $70 \%$ de las mutaciones del C-KIT están localizadas en el exón 11, del $10 \%$ al $15 \%$ en el exón 9 , y menos frecuente en los exones 13 y 17. Las mutaciones del receptor A del factor de crecimiento derivado de plaquetas (PDGFRA) se encuentran usualmente en el exón 18 (13). La mayoría de las mutaciones de los GIST gástricos corresponde al exón 11. Solo 2 de nuestros pacientes tuvieron un estudio mutacional y correspondían al exón 11.

La apariencia endoscópica es de una lesión subepitelial con o sin ulceración. También endoscópicamente se puede tener la sensación de compresión extrínseca con un mínimo compromiso de la mucosa. Estos tumores pueden tener tamaños grandes con crecimiento importante y mayor demanda de suplencia vascular, lo que origina ulceración central de la lesión (12).

Varios estudios han reportado la existencia de lesiones conocidas como micro-GIST, que corresponden a lesiones menores de $1 \mathrm{~cm}$, las cuales son descubiertas en estudios de patología producto de gastrectomías por cáncer (35\%) o en estudios de autopsias de pacientes mayores de 50 años $(22,5 \%)$. Con la endoscopia digestiva alta se ha incrementado el diagnóstico de mayor número de lesiones o tumores subepiteliales. Varios estudios han demostrado que son más comunes de lo que se había presumido. Por esta razón, es muy importante para un endoscopista estar familiarizado con el manejo de esta patología (15).

Las lesiones gástricas se presentan como micro-GIST (lesiones menores de $1 \mathrm{~cm}$ ), mini-GIST (de 1 a $2 \mathrm{~cm}$ ) y los GIST clínicamente relevantes (sintomáticos o mayores de 2 $\mathrm{cm}$ ). Los 2 primeros son indolentes y no progresan clínicamente de forma significativa. La mayoría de los micro-GIST parece ser menos activa desde el punto de vista mitótico y tienen diferentes mutaciones comparadas con los GIST clínicamente relevantes. En un análisis que compara 101 GIST $<2 \mathrm{~cm}$, con 170 lesiones $>2 \mathrm{~cm}$, la mayoría de los tumores menores de $1 \mathrm{~cm}$ no tenía actividad mitótica (14).

La mayoría de las lesiones subepiteliales se identifica incidentalmente por endoscopia por indicación de otras causas, tamización, chequeos médicos, controles endoscópicos de otra causa, entre otros. Estas lesiones incluyen GIST, leiomiomas, lipomas, schwannomas, páncreas ectópico o duplicación quística. Estas lesiones no pueden ser diagnosticadas hasta no tener el examen histológico; sin embargo, obtener una muestra de estas lesiones por biopsia convencional endoscópica es difícil. Por este motivo, la punción con aguja fina con endosonografía es la mejor forma de obtener muestras de tejido para el diagnóstico patológico (14).

Es bien conocido que algunas lesiones gástricas se presentan en la práctica clínica como tumores subepiteliales. Casi la mitad de estas lesiones corresponde a un GIST después de la resección quirúrgica (14).

La biopsia por aspiración con aguja fina (ACAF) por endosonografía ha mostrado recientemente la confirmación histológica de estas lesiones y sugiere una estrategia de manejo. Sin embargo, es difícil obtener muestras de tejido adecuadas de tumores pequeños y de difícil localización. Los parámetros endoscópicos y endosonográficos que incluyen el aumento en el tamaño del $25 \%$ de la lesión, bordes externos irregulares, espacios quísticos y alteración de la ecogenicidad son indicativos de cambios malignos (15).

Este tipo de lesiones de localización gástrica pueden ser clasificadas en varios tipos (15):

- tipo I: lesión con una relación muy pequeña con la muscular propia y protruye a la luz gástrica, de forma similar a un pólipo;

- tipo II: lesión con un contacto amplio con la muscular propia y también protruye a la luz gástrica;

- tipo III: lesión localizada en la parte media de la pared gástrica, dentro de la muscular propia; 
- tipo IV: lesión que protruye sobre la serosa del estómago con mayor compromiso extragástrico.

Las lesiones menores de $2 \mathrm{~cm}$ pueden ser seguidas con control endosonográfico de forma segura y anual. También deben ser resecadas si se determinan cambios en el tamaño y en las características endosonográficas. Para lesiones de mayor tamaño, es necesario tener un diagnóstico histológico por los diagnósticos diferenciales. Si no es posible la punción con aguja fina por endosonografía, la resección primaria quirúrgica debe ser la mejor alternativa (15).

La remoción quirúrgica es el tratamiento primario para una lesión localizada. El objetivo es la resección completa de la lesión con márgenes negativos (15). Es muy importante la no ruptura de la pseudocápsula del tumor por el peligro de diseminación de la enfermedad, lo que establece un mal pronóstico (15).

Dependiendo de la localización de la lesión y su tamaño, está indicado el tipo de tratamiento quirúrgico. Las resecciones amplias o en cuña son las preferidas para la mayoría de las lesiones, pero en ocasiones debe pensarse en gastrectomías parciales, subtotales o totales. La cirugía con resección por laparoscopia, la cual es menos invasiva que la cirugía tradicional, ha demostrado resultados similares en términos de eficacia, seguridad y estancia hospitalaria (16). Algunas guías sugieren que las lesiones menores de $5 \mathrm{~cm}$ son las preferidas para el manejo laparoscópico. Sin embargo, los abordajes laparoscópicos han expandido sus indicaciones para lesiones de mayor tamaño, pero lo importante es la experticia en el manejo oncológico, lo que evita la ruptura durante la resección y obtiene adecuados márgenes libres de lesión (17).

Luego, el manejo quirúrgico depende de su tamaño, de su localización y de la experiencia del operador. Se pueden practicar resecciones endoscópicas, resecciones quirúrgicas abiertas o por laparoscopia, resecciones con procedimientos combinados de laparoscopia y ayuda endoscópica (cirugía cooperativa), y cirugía transgástrica $(15,18)$.

En una revisión sistemática y metaanálisis en el que se compara la cirugía laparoscópica frente a la cirugía abierta en el manejo de los GIST gástricos, en 189 estudios se encontró que no existía diferencia entre el tiempo quirúrgico, los eventos adversos, la pérdida de sangre intraoperatoria, la supervivencia global y las cifras de recurrencia. Este trabajo concluye que la cirugía laparoscópica es segura y efectiva para el manejo de los GIST gástricos y está asociada con una menor estancia hospitalaria (19).

Para lesiones muy grandes y cerca de la unión esofagogástrica o el píloro, la mejor alternativa es la gastrectomía total o subtotal, evitando las deformidades y la mala evacuación gástrica. El tamaño de la lesión, su ubicación y patrón de crecimiento determinan tanto la necesidad de utilizar endoscopia intraoperatoria como el tipo de resección. En lesiones tipo IV, la identificación solo por laparoscopia es suficiente y usualmente la resección en cuña o exogástrica con sutura mecánica lineal ofrece una rápida alternativa con un adecuado margen de resección (19-21). Por el contrario, las lesiones tipo I ubicadas en la pared anterior pueden requerir identificación endoscópica y, según su tamaño, resecciones transgástricas incluida la superficie serosa, en la que se encuentra su base de implantación con el cierre subsecuente del defecto gástrico (22). Los tumores ubicados en la pared posterior se pueden resecar también a través de una gastrotomía en la pared anterior con la realización de sutura mecánica desde el interior del estómago con el cierre de la gastrotomía ulterior; de lo contrario, se puede sacrificar tejido gástrico sano, lo que puede ocasionar deformidades que eventualmente se traducen en un inadecuado tránsito gástrico. Las lesiones del fondo o aquellas ubicadas en la curvatura mayor pueden resecarse con técnica exogástrica. La técnica intragástrica utilizando trócares transabdominales transgástricos está diseñada para lesiones subcardiales menores de $4 \mathrm{~cm}$, excepto las de la pared anterior por la dificultad técnica presentada con la subsecuente extracción de la lesión de forma peroral (11, 18-20, 22, 23).

Otra alternativa de manejo para estas lesiones subepiteliales es la resección endoscópica con tunelización submucosa. Esta técnica tiene ventajas sobre la disección submucosa en cuanto a mantener la integridad de la mucosa y la submucosa, que promueve o facilita la cicatrización de la herida y reduce el riesgo de infección pleural y abdominal. Esta técnica debe ser realizada por endoscopistas con experiencia y está indicada en lesiones menores de $3 \mathrm{~cm}$ y preferiblemente de localización no profunda de la pared muscular por el riesgo de lesionar la serosa (24).

Este trabajo tiene las limitaciones de ser retrospectivo y tener un número pequeño de casos, pero con la incidencia de esta patología puede ser representativo y significativo.

\section{CONCLUSIONES}

De 31 pacientes recolectados con GIST gástrico durante 10 años, la mayoría (67\%) se manejó con cirugía abierta; sin embargo, se utilizó la laparoscopia de forma segura como alternativa y, de este modo, se brindaron los beneficios de la cirugía de invasión mínima con seguridad desde el punto de vista oncológico. La localización más frecuente en el estómago fue el cuerpo, con 20 casos. El dolor abdominal, el sangrado y la masa abdominal fueron los síntomas más relevantes. La mediana de seguimiento estuvo por encima de los 40 meses y más del $50 \%$ se encontraba vivo. 
Al momento del corte del estudio, solo 2 pacientes habían muerto. Se revisaron los conceptos más relevantes en relación con el manejo del GIST gástrico, que son de interés para cualquier endoscopista.

\section{Fuentes de financiación}

Instituto Nacional de Cancerología.

\section{REFERENCIAS}

1. Wente MN, Büchler MW, Weitz J. Gastrointestinale Stromatumoren (GIST). Chirurgische Therapie. Chirurg. 2008;79(7):638-43. https://doi.org/10.1007/s00104-008-1527-5

2. Joensuu H, Hohenberger P, Corless CL. Gastrointestinal stromal tumour. Lancet. 2013;382(9896):973-83. https://doi.org/10.1016/S0140-6736(13)60106-3

3. McDonnell MJ, Punnoose S, Viswanath YKS, Wadd NJ, Dhar A. Gastrointestinal stromal tumours (GISTs): an insight into clinical practice with review of literature. Frontline Gastroenterol. 2017;8(1):19-25. https://doi.org/10.1136/flgastro-2015-100670

4. Miettinen M, Sobin LH, Lasota J. Gastrointestinal stromal tumors of the stomach: a clinicopathologic, immunohistochemical, and molecular genetic study of 1765 cases with long-term follow-up. Am J Surg Pathol. 2005;29(1):52-68. https://doi.org/10.1097/01.pas.0000146010.92933.de

5. Yacob M, Inian S, Sudhakar CB. Gastrointestinal Stromal Tumours: Review of 150 Cases from a Single Centre. Indian J Surg. 2015;77(Suppl 2):505-10. https://doi.org/10.1007/s12262-013-0899-z

6. Machairas A, Karamitopoulou E, Tsapralis D, Karatzas T, Machairas N, Misiakos EP. Gastrointestinal stromal tumors (GISTs): an updated experience. Dig Dis Sci. 2010;55(12):3315-27. https://doi.org/10.1007/s10620-010-1360-9

7. Folgado Alberto S, Sánchez P, Oliveira M, Cuesta L, Gomes F, Figueiredo A, et al. Tumores del estroma gastrointestinal. Estudio retrospectivo de 43 casos. Rev Esp Enferm Dig. 2008;100(11):696-700. https://doi.org/10.4321/S1130-01082008001100005

8. Vargas C A, Cardona A, Carranza H, Otero Jorge Miguel, Reveiz Ludovic, Ospina Édgar, et al. Tumor estromal Gastrointestinal (GISTs): experiencia en dos instituciones hospitalarias de Bogota DC Colombia. Rev Col Gastroenterol 2008;23(3):213-223.

9. Oliveros R, Quintero P, Mesa J, Mesa JA. Tumor estromal gastrointestinal: revisión del 2000 al 2008. Rev Col Cancerol. 2011:15(4):202-211. https://doi.org/10.1016/S0123-9015(12)70050-1

10. Flores-Funes D, Lirón-Ruiz RJ, Pérez-Guarinos CV, Martín-Lorenzo JG, Torralba-Martínez JA, GiménezBascuñana A, et al. Clinical and pathological features of gastrointestinal stromal tumors (GIST) in a single institution: A descriptive study and review of the literature. Cir
Esp. 2017;95(7):391-396. https://doi.org/10.1016/j.ciresp.2017.06.005

11. Bennett JJ, Rubino MS. Gastrointestinal stromal tumors of the stomach. Surg Oncol Clin N Am. 2012;21(1):21-33. https://doi.org/10.1016/j.soc.2011.09.008

12. Maor Y, Avidan B, Melzer E, Bar-Meir S. Long-term clinical outcome of patients with gastric gastrointestinal stromal tumors. Dig Dis Sci. 2010;55(10):2893-8. https://doi.org/10.1007/s10620-009-1107-7

13. Kim MC, Yook JH, Yang HK, Lee HJ, Sohn TS, Hyung WJ, et al. Long-Term Surgical Outcome of 1057 Gastric GISTs According to 7th UICC/AJCC TNM System: Multicenter Observational Study From Korea and Japan. Medicine (Baltimore). 2015;94(41):e1526. https://doi.org/10.1097/MD.0000000000001526

14. Nishida T, Goto O, Raut CP, Yahagi N. Diagnostic and treatment strategy for small gastrointestinal stromal tumors. Cancer. 2016;122(20):3110-3118. https://doi.org/10.1002/cncr.30239

15. Kim HH. Endoscopic treatment for gastrointestinal stromal tumor: Advantages and hurdles. World J Gastrointest Endosc. 2015;7(3):192-205. https://doi.org/10.4253/wjge.v7.i3.192

16. Daigle C, Meneghetti AT, Lam J, Panton ON. Laparoscopic management of gastrointestinal stromal tumours: review at a Canadian centre. Can J Surg. 2012;55(2):105-9. https://doi.org/10.1503/cjs.031410

17. Judson I, Bulusu R, Seddon B, Dangoor A, Wong N, Mudan $S$. UK clinical practice guidelines for the management of gastrointestinal stromal tumours (GIST). Clin Sarcoma Res. 2017;7:6. https: / / doi.org/10.1186/s13569-017-0072-8

18. Tan Y, Tan L, Lu J, Huo J, Liu D. Endoscopic resection of gastric gastrointestinal stromal tumors. Transl Gastroenterol Hepatol. 2017;2:115. https://doi.org/10.21037/tgh.2017.12.03

19. Pelletier JS, Gill RS, Gazala S, Karmali S. A Systematic Review and Meta-Analysis of Open vs. Laparoscopic Resection of Gastric Gastrointestinal Stromal Tumors. J Clin Med Res. 2015;7(5):289-96. https://doi.org/10.14740/jocmr1547w

20. Loureiro Mde P, Almeida RA, Claus CM, Bonin EA, CuryFilho AM, Dimbarre D, et al. Laparoscopic resection of gastrointestinal stromal tumors (GIST). Arq Bras Cir Dig. 2016;29(1):1-4. https://doi.org/10.1590/0102-6720201600010001 
21. Ismael H, Ragoza Y, Caccitolo J, Cox S. Optimal management of GIST tumors located near the gastroesophageal junction: Case report and review of the literature. Int J Surg Case Rep. 2016;25:91-6.

https://doi.org/10.1016/j.ijscr.2016.06.006

22. Ntourakis D, Mavrogenis G. Cooperative laparoscopic endoscopic and hybrid laparoscopic surgery for upper gastrointestinal tumors: Current status. World J Gastroenterol. 2015;21(43):12482-97.

https://doi.org/10.3748/wjg.v21.i43.12482
23. Borráez B, Arévalo C, Montoya J, Buitrago DA, Buelvas N, Oliveros R, et al. Cirugía gástrica laparoendoscópica cooperativa. Rev Argent Cirug 2018;110(1):17-22. https://doi.org/10.25132/raac.v110.n1.1337.es

24. Ye LP, Zhang Y, Mao XL, Zhu LH, Zhou X, Chen JY. Submucosal tunneling endoscopic resection for small upper gastrointestinal subepithelial tumors originating from the muscularis propria layer. Surg Endosc. 2014;28(2):524-30.

https://doi.org/10.1007/s00464-013-3197-8 